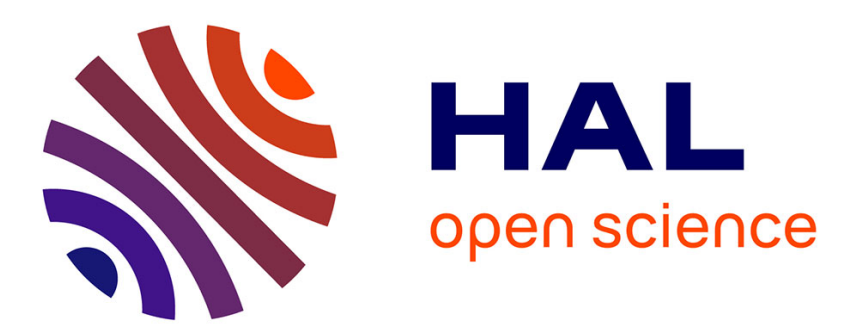

\title{
La peur du déclin économique face à l'épuisement des ressources naturelles, de W. Stanley Jevons à Herbert S. Jevons (1865-1915)
}

Antoine Missemer

\section{- To cite this version:}

Antoine Missemer. La peur du déclin économique face à l'épuisement des ressources naturelles, de W. Stanley Jevons à Herbert S. Jevons (1865-1915). Revue Economique, 2015, 66 (5), pp.825-842. 10.3917/reco.665.0825 . hal-01215291

\author{
HAL Id: hal-01215291 \\ https://hal.science/hal-01215291
}

Submitted on 17 Mar 2017

HAL is a multi-disciplinary open access archive for the deposit and dissemination of scientific research documents, whether they are published or not. The documents may come from teaching and research institutions in France or abroad, or from public or private research centers.
L'archive ouverte pluridisciplinaire HAL, est destinée au dépôt et à la diffusion de documents scientifiques de niveau recherche, publiés ou non, émanant des établissements d'enseignement et de recherche français ou étrangers, des laboratoires publics ou privés.

\section{(ㅇ)(1) $\$$}

Distributed under a Creative Commons Attribution - NonCommercial - NoDerivatives| 4.0 


\title{
LA PEUR DU DECLIN ECONOMIQUE FACE A L'EPUISEMENT DES RESSOURCES NATURELLES, DE W. STANLEY JEVONS A HERBERT S. JEVONS (1865-1915)
}

\begin{abstract}
Antoine MisSEMER ${ }^{*}$
Reference (to cite the paper):

MiSSEMER, Antoine. 2015. « La peur du déclin économique face à l'épuisement des ressources naturelles, de W. Stanley Jevons à Herbert S. Jevons (1865-1915) ». Revue économique, vol. 66, p. 825-842.
\end{abstract}

[http://dx.doi.org/10.3917/reco.665.0825]

For the correct pagination, see the published version.

\begin{abstract}
La prospérité inédite de la Grande-Bretagne au XIX ${ }^{\mathrm{e}}$ siècle se trouve, dans les années 1860 , sous une menace restée longtemps invisible : l'épuisement des mines de charbon. C'est W. Stanley Jevons qui, le premier, souligne avec vigueur les risques que présente cet épuisement pour l'avenir économique de son pays. Cinquante ans plus tard, en 1915 exactement, son fils, Herbert S. Jevons, prolonge son analyse dans des directions multiples. Si ces deux auteurs s'accordent pour indiquer que l'épuisement des ressources pose un problème de coût de production plus que de pénurie, tous deux ne mettent pas en évidence les mêmes voies de sortie pour éviter le déclin industriel. Cette peur du déclin reste néanmoins une caractéristique forte de la pensée économique britannique du tournant du $\mathrm{XX}^{\mathrm{e}}$ siècle.
\end{abstract}

\footnotetext{
* [In 2015] Centre Walras-Pareto, University of Lausanne, Internef, CH-1015 Lausanne-Dorigny \& Triangle, University of Lyon 2, ISH, 14 av. Berthelot, F-69007 Lyon. E-mail address: antoine.missemer@univ-lyon2.fr

Je tiens à remercier les participants aux séminaires «Les après-midi d'économie et de philosophie » de Phare, «Le déclin économique » de Triangle, et «Histoire des idées et philosophie économiques » du Centre Walras-Pareto. Merci en particulier à Élodie Bertrand, Victor Bianchini, Maxime Desmarais-Tremblay, Jean-Baptiste Frery, Marion Gaspard, Arnaud Orain, Jean-Pierre Potier et Shirine Sabéran. Merci enfin aux éditeurs et rapporteurs pour leurs suggestions précieuses. Toute erreur ou omission demeure de mon unique responsabilité.
} 


\section{Introduction}

L'ouvrage The Coal Question (1865, $2^{\mathrm{e}}$ éd. 1866) de William Stanley Jevons fait figure, rétrospectivement, d'acte de naissance de l'analyse économique des ressources naturelles épuisables (Missemer [2012], p. 98-100 ; [2014], p. 63-97). Cet acte de naissance est le fruit historique de deux évolutions importantes. Une première évolution a trait à l'histoire des faits, le secteur minier occupant une place toujours plus grande dans le développement industriel et invitant les économistes à tenir compte des contraintes minières dans leurs prospectives. Une deuxième évolution a trait à l'histoire des idées, la prise en considération des ressources du sous-sol dans les raisonnements économiques s'étant développée peu à peu, dans le monde anglo-saxon, à partir des travaux de David Ricardo ([1821], p. 46-47), de John R. McCulloch [1830], [1835], de Nassau Senior [1836] et de John Stuart Mill [1848] ${ }^{1}$. The Coal Question apparaît à la fois comme une concrétisation des inspirations qui l'ont précédé, et comme un point de départ pour une analyse précise et monographique des ressources minières.

Cinquante ans après son père, Herbert Stanley Jevons, professeur d'économie à l'Université d'Allahabad (Inde), passé également par l'Université du Pays de Galles de Cardiff, choisit lui aussi de consacrer une partie de ses recherches à l'industrie charbonnière britannique. Il dresse ainsi, dans The British Coal Trade [1915], une vaste monographie des méthodes et des pratiques minières. Prolongeant le travail de son aîné mais adoptant par endroits des positionnements différents, H.S. Jevons apparaît comme une figure importante des débats miniers du début du $\mathrm{XX}^{\mathrm{e}}$ siècle $^{2}$.

La situation économique britannique dans les années 1860 est celle d'une prospérité sans précédent. Dès les premières décennies du $\mathrm{XIX}^{\mathrm{e}}$ siècle, l'économie britannique expérimente une importante croissance de sa production, qu'Angus

\footnotetext{
${ }^{1}$ Remarquons que, dans le monde francophone, JeanBaptiste Say [1828] et Antoine Augustin Cournot [1863] ont, eux aussi, contribué à l'essor des débats sur l'épuisement des ressources naturelles.

${ }^{2}$ Bien que les deux Jevons adoptent des positions proches dans leurs travaux respectifs de théorie économique (H.S. Jevons [1905] ; W.S. Jevons [1871]), l'accent ne sera pas mis ici sur la constitution et les évolutions de ce corpus marginaliste.
}

Maddison chiffre à plus de $2 \%$ par an entre 1820 et 1870 , des niveaux qui, historiquement, ne seront atteints à nouveau qu'après la Seconde Guerre mondiale (Maddison [2006], p. 187). À l'aube du dernier tiers du XIX ${ }^{\mathrm{e}}$ siècle, la Grande-Bretagne occupe une place prépondérante sur les marchés internationaux, d'une part, en raison de son dynamisme économique, et d'autre part, en raison de son réseau commercial garanti par un empire colonial vaste. La Grande-Bretagne et l'Irlande forment ainsi, en 1870, un ensemble disposant d'un produit intérieur brut supérieur de $40 \%$ à celui des autres grands pays européens, comme l'Allemagne et la France (Maddison [2006], p. 184). Au-delà de ces chiffres d'ensemble, la prospérité britannique est caractérisée, selon les observateurs de l'époque, par quatre traits principaux. 1) La première caractéristique concerne bien entendu la production de richesses qui, comme on l'a vu, est, au milieu du $\mathrm{XIX}^{\mathrm{e}}$ siècle, un pilier de la suprématie britannique. 2) La deuxième caractéristique a trait au domaine démographique. Pour W.S. Jevons, notamment, une nation prospère est une nation qui dispose d'une large population en croissance. Avec plus de 31 millions d'habitants en $1870(+50 \%$ depuis 1820$)$, le Royaume-Uni répond à ce critère (Maddison [2006], p. 183). 3) Troisième caractéristique de la prospérité britannique, la force militaire est un aspect mis en évidence là encore par W.S. Jevons ([1866a], p. 348). Intimement liée à la puissance économique, la sécurisation des voies maritimes apparait, au milieu du XIX ${ }^{\mathrm{e}}$ siècle, comme un gage de stabilité pour garantir à la population une liberté d'action, aussi bien dans la vie de tous les jours que dans la mise en place de projets industriels et commerciaux vastes. 4) Enfin, l'ère victorienne fait figure, pour les intellectuels du $\mathrm{XIX}^{\mathrm{e}}$ siècle, de période faste pour la créativité artistique et le développement du savoir scientifique. Ces quatre caractéristiques dénotent une vision du progrès, chez ces observateurs, qui se construit de manière pragmatique, à partir des réalités observées dans la société victorienne. Pour eux, le déclin économique serait alors à définir, a contrario, comme la perte d'une ou de plusieurs de ces caractéristiques essentielles. Et dans la mesure où la production de richesses, l'horizon démographique, la puissance militaire et l'aisance intellectuelle et artistique dépendent tous, directement ou indirectement, du développement économique, ce développement devient le sujet principal de préoccupation. Que devient-il dans un contexte d'épuisement des 
ressources minières, levier majeur de l'essor industriel ? Cet épuisement des ressources n'annonce-t-il pas le déclin futur de l'économie britannique ? Ce sont les questions que se posent W.S. Jevons et H.S. Jevons, à cinquante ans d'intervalle, selon des modalités à la fois proches et contrastées.

L'objectif de la présente contribution est de souligner les transformations de la peur du déclin économique face à l'épuisement des ressources naturelles, dans une tradition britannique imprégnée par le souhait d'un développement industriel continu. Les travaux de W.S. Jevons et de H.S. Jevons sont, à cet égard, représentatifs, car The Coal Question fait figure de point de départ dans cette tradition et parce que The British Coal Trade apporte un regard relativement complet sur les évolutions de la question minière dans les dernières décennies du $\mathrm{XIX}^{\mathrm{e}}$ siècle et au début du $\mathrm{XX}^{\mathrm{e}}$ siècle. L'étude menée ici tient en particulier à souligner les apports de H.S. Jevons, rarement mobilisés en histoire de la pensée économique et en économie de l'environnement et des ressources naturelles. L'enjeu sera de mesurer la manière dont l'épuisement des ressources constitue un élément structurant de la vision portée par certains économistes, au tournant du $\mathrm{XX}^{\mathrm{e}}$ siècle, sur l'avenir des sociétés industrielles.

Pour mener à bien ce projet, nous débuterons par une exposition de la dépendance énergétique de l'économie britannique au milieu du XIX ${ }^{\mathrm{e}}$ siècle. Nous verrons alors comment W.S. Jevons, comme H.S. Jevons, estiment que l'épuisement des ressources pose des problèmes de coûts avant de poser des problèmes de pénurie physique (section 1). Dans un deuxième temps, nous étudierons l'articulation entre la problématique de l'épuisement et les thèses déclinistes, en identifiant les canaux de transmission pouvant relier la dépendance énergétique et la fragilisation de l'industrie britannique (section 2). Enfin, nous mettrons en exergue les voies de sortie décrites à l'époque pour tenter de juguler la menace du déclin. Nous soulignerons alors que W.S. Jevons et H.S. Jevons n'adoptent pas, sur ce point, la même perspective; cette différence s'expliquant certainement par des évolutions historiques marquées entre les années 1860 et le début du XXe siècle (section 3 ).

\section{Une prospérité menacée par la dépendance énergétique}

\section{L'essor du secteur énergétique et minier}

$\mathrm{Au}$ crépuscule du $\mathrm{XVIII}^{\mathrm{e}}$ siècle, les premières activités industrielles, et la diffusion du chauffage domestique entraînent une augmentation importante de la consommation de bois, en particulier en Grande-Bretagne. Pour palier le renchérissement du prix de ce combustible, les sociétés industrielles se mettent à exploiter de nouvelles ressources, tirées $\mathrm{du}$ sous-sol. Rendu possible par l'avènement d'innovations majeures, à l'instar de la machine à vapeur développée par Watt en 1784, l'usage généralisé $\mathrm{du}$ charbon engendre alors une augmentation rapide et continue de la production de minerai dans la première moitié du $\mathrm{XIX}^{\mathrm{e}}$ siècle. Entre 1800 et 1830, l'Angleterre voit son extraction charbonnière multipliée par deux (Galloway [1882], p. 201). Sur une plus longue période, les chiffres sont particulièrement évocateurs : entre 1775 et 1865 , la production britannique de charbon passe de 8,9 millions de tonnes à 102,3 millions de tonnes (Church [1986], p. 86 ; Flinn [1984], p. 26) ${ }^{3}$.

Les rapports entretenus entre monde minier et industrie atteignent, au $\mathrm{XIX}^{\mathrm{e}}$ siècle, un niveau d'imbrication et d'interdépendance significatif. Le charbon n'est pas seulement un combustible parmi d'autres, il devient le combustible incontournable, « le pain quotidien» de l'industrie (Simonin [1867], p. 5). Sans charbon, le développement industriel, et donc économique, paraît impossible. Cette dépendance ne concerne pas seulement l'industrie lourde, où le minerai est consommé en abondance (Passet [2010], p. 153), il concerne toutes les branches susceptibles d'utiliser des machines (Outram et Fishman [2006], p. 19; Robine [1990], p. 374). Dans son rapport sur la situation des houillères en 1868, Amédée Burat explique ainsi que c'est l'ensemble de la prospérité anglaise qui repose sur le charbon :

La richesse houillère de l'Angleterre est la base réelle de sa suprématie industrielle et commerciale. Ses manufactures ont été créées et se développent grâce au bas prix de la houille; c'est la houille qui fournit à sa navigation maritime 10 millions de tonnes à exporter et

\footnotetext{
${ }^{3}$ La continuité des séries statistiques entre les travaux de Church et de Flinn nous permet d'associer leurs deux estimations dans le temps long.
} 
lui ouvre ainsi toutes les combinaisons de fret nécessaires à son trafic. ([1869], p. 8-9)

Associés, secteur minier et secteur industriel deviennent les piliers du développement économique. En témoignent les chiffres rassemblés par Brian R. Mitchell sur les deux premiers tiers du $\mathrm{XIX}^{\mathrm{e}}$ siècle : alors que la production industrielle et minière représente $23,4 \%$ de la production britannique totale en 1801 , elle en représente $36,5 \%$ en 1861, des chiffres qui mettent en évidence un accroissement d'autant plus impressionnant que la production britannique dans son ensemble a triplé au cours de cette période (Mitchell [1988], p. 822) . Nul besoin d'insister davantage sur ces faits stylisés pour mesurer combien l'économie britannique, la plus en avance au milieu du XIX $\mathrm{X}^{\mathrm{e}}$ siècle, entretenait une dépendance forte avec la santé du secteur énergétique et minier. Dépendance directe en raison du poids du charbon dans l'activité économique globale. Dépendance indirecte en raison de l'importance du secteur industriel, lui-même dépendant de l'activité minière.

\section{L'épuisement des ressources naturelles, une contrainte économique}

En 1865, pour W.S. Jevons, la Révolution industrielle et ses effets sur l'accroissement des richesses expliquent l'essentiel de la réussite économique de la Grande-Bretagne. Or, cette Révolution industrielle a été rendue possible grâce à l'exploitation d'un charbon de bonne qualité, et bon marché. Ce charbon est devenu «la pièce maîtresse de l'industrie britannique " (W.S. Jevons [1868], p. 29). Il s'agit pourtant d'une ressource naturelle qui a une caractéristique fondamentale : sa finitude. Alors qu'une économie agricole peut fonctionner sur la base de récoltes régulières, bien que variables d'une année à l'autre, une économie industrielle repose sur l'extraction irréversible de certains minerais. Une mine épuisée l'est une fois pour toutes. En prenant conscience des conséquences économiques de l'épuisement des ressources énergétiques, W.S. Jevons rend compte de la dépendance énergétique croissante que nous avons soulignée plus haut :

\footnotetext{
${ }^{4}$ Nous calculons ces ratios à partir des données de Mitchell.
}

[...] the power and usefulness of coal is felt in every branch of industry, and in almost every operation which we carry on. ([1868], p. 29)

Cette dépendance énergétique est apparue au grand jour lors de la crise des années 1872-1873, lorsque le renchérissement du minerai s'est diffusé rapidement dans l'ensemble des coûts de production des diverses marchandises industrielles (W.S. Jevons [1875], p. 37). Ce soubresaut historique est intervenu pour W.S. Jevons en tant que signe précurseur de difficultés à venir. Dans la mesure où le charbon est une ressource épuisable, fonder l'ensemble d'un appareil productif sur son seul usage est un risque, risque que les Britanniques ont choisi de prendre afin d'alimenter une dynamique économique prometteuse dans un premier temps, mais promise à un déclin par la suite. En 1897, alors qu'il préside la Royal Statistical Society, Leonard H. Courtney se fait le porte-parole des craintes de W.S. Jevons en donnant l'exemple de la province de Cornwall qui, pendant longtemps, a été florissante grâce à son activité d'extraction du cuivre. Lorsque les gisements se sont épuisés, la région a connu une crise majeure, s'est appauvrie et a vu sa population décroître. Courtney prédit alors, trente ans après W.S. Jevons, que le destin de la Grande-Bretagne tout entière pourrait s'apparenter à celui de la province de Cornwall si les mines de charbon venaient à s'épuiser (Courtney [1897], p. 131). Cet exemple illustre la prégnance de la peur du déclin parmi bon nombre d'observateurs britanniques à la fin du $\mathrm{XIX}^{\mathrm{e}}$ siècle. Et il met en évidence le point d'achoppement que constitue la problématique de l'épuisement des ressources naturelles dans ce contexte.

L'épuisement des ressources peut être conçu sous deux angles distincts : un angle géophysique et un angle économique. Le premier concerne simplement les réserves disponibles. L'étude des ressources naturelles est alors essentielle- ment technique, à l'aide des connaissances et des méthodes des sciences naturelles. Comme l'ont déjà souligné certains observateurs (voir, par exemple, White [1991], p. 64 ; [2004], p. 243), avec The Coal Question, W.S. Jevons déplace le spectre d'analyse de la question géophysique à une question purement économique. Il sera repris sur ce point par son fils. Ce deuxième angle ne vise plus à estimer les réserves de charbon encore disponibles, mais à se concentrer sur deux éléments essentiels : 
d'une part, l'accessibilité économique, matérialisée par la rentabilité des gisements face aux contraintes de coûts subies par les exploitants (W.S. Jevons [1866a], p. 82-84; [1868], p. 33 ; H.S. Jevons [1915], p. 28-29, 756) et, d'autre part, l'étude du côté demande du commerce de minerai, cette demande évoluant sans cesse (W.S. Jevons [1868], p. 31). Si l'épuisement économique intègre la question de l'épuisement physique, il va également au-delà, en prenant en considération le problème des mécanismes économiques d'ajustement entre une offre soumise à des coûts croissants et une demande exprimant des besoins toujours renouvelés.

La peur du déclin économique se fonde sur les conséquences d'un renchérissement du charbon, devenant plus difficile d'accès et donc plus cher. Si le coût d'extraction du charbon, et donc son prix de vente aux industriels, augmentent, alors l'ensemble des biens produits avec du charbon risquent de voir leurs prix augmenter, ce qui pourrait induire une perte de compétitivité pour la Grande- Bretagne sur les marchés internationaux. L'épuisement des ressources naturelles apparaît ainsi comme un vecteur progressif de déclin économique compris dans un sens relatif et non absolu. Ce qui inquiète, c'est davantage le déclassement compétitif de la Grande-Bretagne (par rapport aux États-Unis, à l'Australie et aux autres pays européens) qu'une quelconque pénurie qui viendrait stopper brutalement l'appareil productif.

\section{Les conséquences de l'épuisement et les modalités du déclin}

\section{Les canaux de transmission de l'épuisement à l'activité économique}

Pendant toute la deuxième moitié du XIX $^{\mathrm{e}}$ siècle, une partie significative (et en croissance) des exportations britanniques sont des exportations de minerais : avec les autres matières premières non agricoles, ces minerais représentent, en 1890, 15\% des exportations de la Grande-Bretagne vers le reste $\mathrm{du}$ monde, contre seulement $7,5 \%$ en 1855 (Mitchell [1988], p. 456-457) ${ }^{5}$. Le succès britannique en la matière s'explique par deux facteurs majeurs. Le premier a trait à la qualité du

\footnotetext{
${ }^{5}$ Ces ratios sont calculés à partir des données de Mitchell.
}

charbon, l'anthracite gallois faisant figure de référence internationale en raison de sa bonne combustion et de son faible niveau d'impureté. Le second est à mettre en relation avec ce que nous nommons aujourd'hui la compétitivité-prix de la Grande-Bretagne. Grâce à une mécanisation importante, un bon savoir-faire, et un ensemble d'infrastructures performantes reliant les bassins de production aux lieux de commercialisation, la Grande-Bretagne se trouve en possession d'un charbon non seulement de qualité, mais aussi vendu à des prix inférieurs à ceux de ses concurrents (Church [1986], p. 774-775). Dans un contexte d'épuisement des mines, ces deux atouts se trouvent menacés. Le charbon de bonne qualité, de moins en moins abondant, doit être recherché dans des zones moins accessibles, et peut présenter des défauts. Quant à la compétitivité-prix, elle peut être mise à mal par le renchérissement de certains coûts de production qui résulterait d'une accessibilité réduite.

De là apparaissent, chez W. Stanley Jevons, ce que nous identifions ici comme quatre canaux de transmission principaux entre l'épuisement des ressources et la dégradation de la situation économique britannique. 1) Le premier de ces canaux concerne précisément le commerce du charbon : si les exportations de minerai diminuent en raison d'une perte de compétitivité-prix, la balance commerciale britannique aura tendance à se dégrader, ce qui sera le signe d'une dégradation de la situation économique. 2) L'épuisement du charbon n'a pas que cet effet direct sur la prospérité, il a également un certain nombre d'effets indirects, liés au rôle stratégique des ressources énergétiques dans les modes de production industriels. Le premier de ces effets indirects concerne la dynamique industrielle prise dans sa globalité. Pour W.S. Jevons, l'industrie fonctionne selon un processus qui consiste, grâce à l'ingéniosité humaine, à mettre au point et à produire de nouveaux produits, des produits qui remplacent parfois les anciens, et dont l'apparition est rendue possible grâce à une utilisation plus performante des ressources énergétiques. Si ces ressources viennent à manquer, il n'est pas certain que la dynamique industrielle puisse perdurer :

With fuel and fire, [...], almost anything is easy. By its aid in the smelting furnace or the engine we have effected, for a century past, those successive substitutions of a better for a worse, a cheaper for a 
dearer, a new for an old process, which advance our material civilization. But when this fuel, our material energy, fails us, whence will come the power to do equal or greater things in the future? (W.S. Jevons [1866a], p. 120)

3) Le troisième canal de transmission, qui constitue un deuxième effet indirect à prendre en considération, concerne toujours le secteur industriel, et a la particularité d'engendrer un effet de levier sur la dégradation de la balance commerciale. Puisque la combustion du charbon est à la base de tous les processus industriels, une hausse du prix du minerai a pour conséquence une hausse du prix de tous les biens manufacturés (W.S. Jevons [1866a], p. 314). Or, dans la seconde partie $\mathrm{du} \mathrm{XIX}^{\mathrm{e}}$ siècle, les biens manufacturés sont une source importante de prospérité pour la GrandeBretagne, d'une part, sur le marché domestique en équipant un nombre croissant de foyers, et, d'autre part, sur les marchés internationaux, par le biais des exportations. Si les biens manufacturés britanniques deviennent trop chers, ils ne pourront plus être exportés, ce qui fragilisera la position de la GrandeBretagne dans le commerce international. 4) À partir de ce mouvement sur la balance commerciale, une autre conséquence de l'épuisement des ressources pourrait peser sur la prospérité, en l'occurrence sur son pilier démographique. En développant une grande industrie, la Grande-Bretagne a délaissé, relativement, le secteur agricole. En 1865, les matières premières non agricoles (minerais) et les biens manufacturés produits par ces intrants représentaient $95 \%$ des exportations britanniques. À l'inverse, la nourriture et les matières agricoles ne représentaient que 5\% des exportations, mais près d'un tiers $(31 \%)$ des importations (Mitchell [1988], p. 456) ${ }^{6}$. Pour nourrir une population croissante, la GrandeBretagne s'est appuyée sur sa puissance commerciale pour exporter des biens manufacturés - en 1870, elle réalise, à elle seule, $40 \%$ des exportations européennes et plus de $20 \%$ des exportations mondiales (Maddison [2006], p. 360) -, et ainsi dégager des moyens financiers pour importer de la nourriture en grande quantité. Ces importations ne sont rendues possibles que par l'intermédiaire des exportations dynamiques résultant de l'activité industrielle. $\mathrm{Si}$ la

\footnotetext{
${ }^{6}$ Ces ratios sont calculés à partir des données de Mitchell.
}

compétitivité britannique venait à baisser en raison $\mathrm{du}$ renchérissement du charbon, le pays ne serait plus en mesure de dégager suffisamment de moyens pour importer la nourriture nécessaire à son développement démographique (W.S. Jevons [1867], p. 24).

Ces différents canaux mis en évidence par W.S. Jevons sont intimement liés au contexte économique dans lequel évoluait l'auteur de The Coal Question. Cinquante ans plus tard, tout en conservant un regard assez pessimiste sur la dépendance énergétique, H.S. Jevons va donner à la peur du déclin économique un visage nouveau, certes lié au secteur énergétique et minier, mais selon des modalités différentes.

\section{Vers d'autres explications du déclin}

La peur du déclin économique se focalise, chez W.S. Jevons, autour de la question de l'épuisement des ressources, comme cause ultime de la dégradation à venir de la compétitivité britannique. H.S. Jevons, lui, garde à l'esprit ce raisonnement, tout en cherchant d'autres éléments qui seraient susceptibles de contrarier la modération des prix miniers, et donc la prospérité industrielle. Il assume, à cet égard, l'émancipation de son discours vis-à-vis de celui de son père :

I want very clearly to show that increased depths of working, and the working of poorer quality and thinner seams, is only one of a number of factors affecting the cost of mining and marketing the coal; because in his great work on "The Coal Question" my father did not, I think, give sufficient weight to these other factors. (H.S. Jevons [1915], p. 758)

Parmi les autres facteurs à prendre en considération, le coût du travail occupe une place centrale, surtout lorsque la mécanisation et la complexification des processus d'extraction nécessitent une main-d'œuvre plus qualifiée qu'auparavant. Ici apparaît une première distinction entre les travaux de W.S. Jevons et d'H.S. Jevons, une distinction qui s'explique en partie par des évolutions historiques. Dans les années 1860, l'extraction minière est jugée comme techniquement possible jusqu'à des profondeurs d'environ 4000 pieds (environ 1200 mètres), grâce à des systèmes de ventilation relativement efficaces, mais qui connaissent des marges de progression encore importantes (W.S. Jevons 
[1866a] ; Galloway [1882]). En 1915, au moment de la publication de The British Coal Trade, cette limite de 4000 pieds reste la plus répandue, bien que des mines soient dorénavant creusées jusqu'à 6000 pieds (environ 1800 mètres), comme H.S. Jevons le remarque ([1915], p. 729). L'extraction $\mathrm{du}$ charbon dans ces profondeurs accrues exige alors des infrastructures de ventilation et de remontée du minerai plus complexes, nécessitant une main-d'œuvre parfois plus qualifiée, et donc plus chère (Hodges [1910], p. 145). À ce renchérissement $\mathrm{du}$ coût $\mathrm{du}$ travail lié à l'accessibilité des ressources s'ajoutent d'autres renchérissements, dus à l'essor de la réglementation minière (à ce sujet, voir Fouquet [2009], p. 13 ; Galloway [1882], p. 230, ou encore Percy [1888], p. 13) et au développement des syndicats (voir Outram [2012b], p. xii-xiii ; Outram [2012c], p. 375-377). Résultat : si les salaires des mineurs ont connu d'importantes variations d'une année sur l'autre jusque dans les années 1890 , ils ont ensuite pris une tendance croissante, augmentant de près de 50\% entre 1897 et 1914 (Mitchell [1988], p. 170) ${ }^{7}$. Une réalité observée par H.S. Jevons, et l'invitant à élargir son regard sur les attaques portées à la compétitivité-prix britannique par rapport aux avertissements de W.S. Jevons centrés sur la seule question des difficultés physiques de production :

The foregoing observations will make it plain how much The Coal Question is in reality a labour question [...]. The miners are now becoming so well organised that they may very well decide that wages shall not fall below a certain minimum [...]. The price of coal in the future is likely to be affected in greater degree by the attitude of labour, and by the cost of meeting stringent legislative requirements, than by the mere cost of deeper working and poorer seams. (H.S. Jevons [1915], p. 760761)

Le coût du travail représente donc un marqueur important de la dépendance énergétique. Si ce coût augmente, l'ensemble du secteur minier, puis l'ensemble des secteurs industriels, verront leurs coûts de production renchéris, au détriment de la compétitivité-prix. Quand on sait que les salaires représentaient, à la fin du $\mathrm{XIX}^{\mathrm{e}}$ siècle, 70 à $80 \%$ des coûts de l'exploitation minière (Outram [2012a], p. 107), force est d'admettre que cette variable avait un effet crucial sur la fixation des prix du charbon.

En cherchant à opérer une mise à jour du travail

\footnotetext{
${ }^{7}$ Ratio calculé à partir des données de Mitchell.
}

de son père, H.S. Jevons réalise, en 1915, que son aîné avait vu juste quant au déclassement compétitif de la Grande-Bretagne (H.S. Jevons [1915], p. 720721). Au niveau international, le secteur énergétique a continué à connaître un essor significatif, la production mondiale de charbon passant de 144 millions de tonnes à 866 millions de tonnes entre 1860 et 1901 (Fernow [1902], p. 421). Certes, la Grande-Bretagne a joué un rôle essentiel dans cet essor. Mais elle n'a pu le jouer seule. L'industrie américaine y a grandement contribué ${ }^{8}$. En 1901, la production américaine de charbon représente déjà, à elle seule, $34 \%$ des volumes mondiaux (Fernow [1902], p. 421). La première puissance charbonnière n'est alors plus la GrandeBretagne, malgré une présence toujours importante dans les échanges internationaux, mais les ÉtatsUnis ${ }^{9}$. Deuxième métamorphose d'ampleur dans le domaine énergétique : l'essor du pétrole. Le premier puits de pétrole mis en activité à des fins industrielles l'a été au cœur des États-Unis, à Titusville (Pennsylvanie), en 1859 (voir, à ce sujet, Copinschi [2012], p. 25). L'absence de pétrole en Grande-Bretagne ${ }^{10}$, face aux abondantes dotations américaines, a aussi joué un rôle dans le déclassement britannique.

Pour expliquer ce déclassement, H.S. Jevons ne limite pas son analyse à une simple répétition des arguments de son père, il cherche également à mettre en exergue d'autres causes que la seule question de la disponibilité des ressources. Il commence par faire référence à la situation géographique des nations qui entrent en concurrence. Parce que les États-Unis constituent un grand marché, ils ont tendance à déplacer le centre névralgique du commerce international, de l'Europe continentale à la façade atlantique. Corrélée à l'apparition de nouveaux acteurs,

\footnotetext{
${ }^{8}$ Si l'Allemagne a également connu un développement spectaculaire à l'époque, W.S. Jevons, comme H.S. Jevons, insistent davantage sur leur partenaire commercial d'outre-Atlantique en raison de la richesse minérale du sous-sol américain, jugée plus menaçante que le développement allemand.

9 Les raisons historiques de ce basculement sont plurielles : abondance des réserves américaines faciles d'accès, et plus grande productivité du travail minier aux États-Unis, surpassant la productivité britannique dès les années 1890 (Mitchell [1988], p. 774).

${ }^{10}$ Les réserves offshore de la mer du Nord, si tant est qu'elles aient été connues, n'étaient pas techniquement exploitables à l'époque.
} 
notamment en Asie avec le Japon, ce déplacement tend à marginaliser, en termes relatifs, la position de la Grande-Bretagne. Avec des délais de livraison plus longs, et des coûts de transport parfois élevés, les produits britanniques perdent en compétitivité, et ce pour des raisons assez lointaines de la seule question de l'accessibilité des ressources (H.S. Jevons [1915], p. 686 et $s q$.). Ensuite, H.S. Jevons évoque l'organisation des affaires aux États-Unis (infrastructures, droit des sociétés, etc.), et affirme que les conditions économiques sont meilleures outre-Atlantique qu'en Europe pour le développement industriel, et pour l'essor entrepreneurial aussi bien sur les marchés domestiques qu'à l'international. Il s'appuie ici notamment sur le développement récent de grandes entreprises américaines :

I do not underrate the probability of serious competition from America, both in the manufacture of crude and finished steel, and in many manufactures, and also in the export of coal; but such serious competition will be due, not mainly to the possession of a cheaper coal supply, but rather to their vastly more efficient business organisation and mechanical appliances. ([1915], p. 767)

H.S. Jevons est donc sensible à la question du déclin britannique, tout comme W.S. Jevons. Les explications qu'il fournit à ce déclin sont cependant plus larges et parfois plus indirectement liées à l'épuisement des ressources que ce que l'on pouvait trouver dans The Coal Question. En tenant compte des évolutions récentes du monde économique (coûts du travail, commerce international) et des systèmes institutionnels, le fils complète, en quelque sorte, l'analyse du père, mais ne perd pas de vue des thèses déclinistes qui, à l'aune de la Première Guerre mondiale, restent un sujet de préoccupation.

\section{Quelles perspectives pour lutter contre le déclin?}

\section{Des solutions technologiques insuffisantes}

Les craintes pesant sur la prospérité de la Grande-Bretagne s'expriment avec une véritable lucidité au tournant du $\mathrm{XX}^{\mathrm{e}}$ siècle, mais ne font pas l'objet d'un simple fatalisme. W.S. Jevons, comme H.S. Jevons, tentent de décrire des voies de sortie qui pourraient permettre à la société britannique, non pas de contrecarrer définitivement son destin, mais de contourner du mieux possible les difficultés à venir. Puisque l'épuisement des ressources énergétiques constitue un obstacle majeur au développement industriel futur, un moyen d'action efficace contre le déclin serait, a priori, de lutter contre cet épuisement en incrémentant dans l'appareil productif des technologies nouvelles, moins gourmandes en ressources. Cette perspective d'ingénierie pour traiter la question de l'épuisement a fait l'objet d'une attention particulière de la part de W.S. Jevons. C'est d'ailleurs dans cette optique que The Coal Question retient le plus l'attention des économistes contemporains. Dans son ouvrage, W.S. Jevons fonde le concept d'effet rebond ([1866a], p. 123-124, 132-133, 136), que l'on retrouve aujourd'hui communément dans la littérature économique (pour un panorama, voir Greening et al. [2000]). Ce concept renvoie à un mécanisme simple : lorsqu'un gain d'efficacité énergétique est réalisé dans un processus économique, ce gain ne se traduit pas par une baisse de l'énergie totale consommée, mais par une hausse. En effet, en étant plus efficace sur le plan énergétique, une nouvelle technologie tend à diminuer le coût de l'énergie, et donc à susciter de nouveaux usages qui viennent annuler les gains initiaux. L'effet rebond est l'outil analytique qui permet à l'auteur de The Coal Question d'écarter les arguments de certains de ses détracteurs qui placent une confiance excessive dans le progrès technique (W.S. Jevons [1868], p. 31). Même si l'industrie britannique était capable d'utiliser le charbon plus efficacement, cela ne tendrait pas à diminuer la pression continue sur l'industrie minière. Pour W.S. Jevons, le charbon est bel et bien utilisé plus efficacement dans les années 1860 qu'au début du XIX ${ }^{\mathrm{e}}$ siècle, mais cela n'a pas pour autant diminué le rythme de croissance de la demande de minerai :

Iron is now made by much less coal than it used to be, yet we use more coal than ever. Engines are better now than they were [...], but that has not cut down our coal consumption; then what is the likelihood that it will do so in the future? ([1867], p. 26)

H.S. Jevons, en 1915, confirme ces observations et souligne, lui aussi, que l'accroissement de l'efficacité énergétique de l'appareil productif, sensible dans les années 1880 et 1890 , ne s'est pas traduit par une baisse de la demande de charbon, même par tête ([1915], p. 746-747). Dans la logique 
exposée ici, l'amélioration de l'appareil productif n'est pas un facteur susceptible de détendre la dépendance énergétique de la Grande-Bretagne envers les ressources minières.

Une autre solution technologique envisageable serait l'utilisation d'une autre ressource que le charbon, pour que la dépendance énergétique n'implique pas une contrainte trop forte sur la production à moyen terme. La substitution du charbon par d'autres ressources est une éventualité mesurée par W.S. Jevons. À cet égard, deux problèmes sont alors soulevés. Le premier concerne une question technique de rendement énergétique : les rendements des ressources alternatives n'apparaissent pas suffisants pour assurer la perpétuation du développement industriel (W.S. Jevons [1866a], p. 156-157, 164). Le deuxième problème soulevé sur la question des substitutions énergétiques concerne une réflexion d'ordre logique. L'électricité, par exemple, présentée dès les années 1860 comme une nouvelle source d'énergie prometteuse, ne peut être un substitut pertinent, et ce pour une raison simple : ce n'est pas une source d'énergie, mais uniquement un véhicule énergétique. En effet, l'électricité doit toujours être produite à partir de quelque chose. Et en l'occurrence, dans la deuxième partie du $\mathrm{XIX}^{\mathrm{e}}$ siècle, elle l'était souvent par combustion du charbon. En 1915, la situation pour H.S. Jevons reste fondamentalement la même. L'électricité, dont l'usage est de plus en plus diffus, notamment dans les mines, est souvent produite avec du charbon à proximité des gisements, pour alimenter ensuite, à plusieurs dizaines de kilomètres de là, les grandes métropoles britanniques (H.S. Jevons [1915], p. 174) ${ }^{11}$.

Restent des énergies dont l'exploitation demeure, pour l'heure, essentiellement conceptuelle, à l'instar de l'énergie solaire. Dans The Coal Question, W.S. Jevons évoque la question et estime que l'énergie solaire sera peut-être, un jour, une solution au problème de l'épuisement des ressources. Il remarque cependant que la problématique du déclin relatif ne sera pour autant pas totale- ment résolue, tout simplement parce que la Grande-Bretagne n'est pas la région du monde la mieux placée pour profiter des rayonnements du soleil (W.S. Jevons [1866a], p. 34-35), argument

\footnotetext{
${ }^{11}$ Le charbon restera la première source de production d'électricité, dans la plupart des pays industrialisés, jusque dans les années 1960 (Fouquet [2009], p.11).
}

avec lequel H.S. Jevons est amplement d'accord ([1915], p. 795-798). D'autres zones géographiques, comme l'Australie ou l'Afrique, devraient, dans le cas d'un usage massif de l'énergie solaire, prendre le pas sur la GrandeBretagne en termes de place stratégique sur les marchés mondiaux. Le déclin relatif de l'Empire britannique ne serait, sous cet angle, pas évité.

L'existence potentielle de substituts au charbon ne convainc donc ni W.S. Jevons, ni H.S. Jevons qui, lui aussi, doute des rendements des autres sources d'énergie parfois présentées (H.S. Jevons [1915], p. 9). Éviter le déclin économique par l'intermédiaire de solutions technologiques n'est donc pas une perspective crédible, du moins à court et moyen termes, pour ces observateurs de la société britannique.

\section{Un contournement du problème par compensation intergénérationnelle}

Pour W.S. Jevons, la crainte du déclin économique, reposant sur l'épuisement des ressources, ne peut être apaisée à l'aide d'instruments techniques. Le seul moyen d'agir pour contrecarrer l'avènement d'un déclin réside dans un contournement du problème, afin d'opérer non sur la cause des dégradations futures, mais sur leurs conséquences. W.S. Jevons met au point un principe qui repose sur une certaine solidarité intergénérationnelle, à partir de présupposés clairs : dans la mesure où le progrès économique et social est une aspiration universelle, il ne peut être réservé à une seule génération. $\mathrm{La}$ justice intergénérationnelle est ainsi conçue par W.S. Jevons comme un droit universel au progrès : chaque génération doit pouvoir bénéficier des mêmes capacités que les autres à voir sa situation économique s'améliorer. $\mathrm{Si}$ les ressources naturelles sont le levier principal de ce progrès, elles doivent être gérées avec responsabilité par ceux qui sont en situation de les exploiter. Cela ne signifie pas, pour W.S. Jevons, que les générations présentes doivent limiter leur usage des ressources. En revanche, les richesses produites à partir de ces ressources doivent être conçues pour participer au développement futur, et pas seulement dans une logique de consommation présente qui pourrait prendre les traits d'un gaspillage. Les richesses produites aujourd'hui doivent donc être orientées vers des investissements en équipements (logements) et en éducation, pour permettre aux 
générations futures de profiter, elles aussi, de la période actuelle de prospérité :

It strikes me that the best way to prepare for future time is by taking every advantage of the present. I do not think that our descendants will blame us if we take proper precautions to use our coal economically, and to get the best possible return for it - that is to say, the most force and the most wealth, and not to burn it needlessly upon waste heaps, as is sometimes done. [...] We must use our wealth as it ought to be used. If we use it in mere luxury and mismanagement, such as in our dockyards, we shall be justly blamed; but if we use it in improving the condition of every one, so far as it can be improved if we use it in providing education, in improving the dwellings; and if we could by any possibility use it so as to do away with pauperism, and to provide libraries and institutions or anything that will increase the power and improve the character of our people, then I think we shall never be blamed for using our coal too fast. (W.S. Jevons [1867], p. 27-28)

Cette orientation signifie une constante lutte contre les gaspillages, mais elle s'apparente également, selon notre lecture, à un principe de compensation intergénérationnelle, où les générations présentes utilisent les ressources énergétiques qu'elles ont en grande quantité, en laissant en contrepartie aux générations futures un ensemble de capitaux, physiques et humains, offrant un dédommagement vis-à-vis du renchérissement à venir des ressources naturelles (W.S. Jevons [1866a], p. 369). Ce mécanisme de compensation fait écho, d'une certaine manière, aux débats de la fin du $\mathrm{XX}^{\mathrm{e}}$ siècle sur la soutenabilité faible (à ce sujet, voir Gowdy et O'Hara [1997] ; Neumayer [1999] ; Pearce et Atkinson [1993]). Il ne couvre néanmoins pas l'entièreté de ces débats dans la mesure où W.S. Jevons n'entend pas l'investissement en capital physique comme la production de n'importe quels biens de production mais comme la construction de logements et d'équipements publics. De plus, si son argumentaire renvoie à une substitution entre ressources naturelles et capitaux artificiels et humains, il n'implique pas ici la redistribution intertemporelle d'une rente, comme on peut le trouver dans les réflexions issues des travaux de John M. Hartwick [1977].

Ce type de raisonnement, W.S. Jevons l'effleure cependant plus clairement lorsqu'il propose, dans la deuxième partie de The Coal Question, un outil singulier pour participer au dédommagement des générations futures, à savoir la réduction de la dette publique ([1866a], p. 364-366). La dette publique a un caractère d'injustice, en ce qu'elle finance des projets pour les générations présentes, alors que son remboursement devra être effectué par les générations futures. Le fardeau de la dette publique vient s'ajouter aux difficultés qui pourraient survenir à la suite de l'épuisement des ressources énergétiques. Cette double peine n'est, pour W.S. Jevons, pas acceptable. L'enjeu essentiel de la lutte contre le déclin économique passe alors par une réduction, voire un effacement, de la dette publique, une entreprise qui nécessite une audace politique dont ses contemporains se doivent de faire preuve ([1866a], p. 364-365).

Nous pouvons noter ici que le raisonnement de W.S. Jevons laisse une zone d'ombre sur les mesures concrètes à mettre en place pour réduire la dette publique. En effet, il n'est pas certain qu'il soit possible de mener simultanément une politique d'investissement dans des infrastructures et dans l'éducation, tout en veillant à réduire l'endettement public. Si W.S. Jevons choisit de laisser aux milieux politiques le soin de mettre au jour ces mesures concrètes ([1866b]), il met en évidence, quoi qu'il en soit, deux principes qui ne sont peutêtre pas conciliables. Par ailleurs, sa conception de la dette publique comme fardeau collectif peut également susciter des interrogations, dans la mesure où les générations présentes ne lèguent pas uniquement des dettes à leurs descendants, mais également des créances. Plus qu'un problème d'épée de Damoclès, la dette publique génère sans doute des effets redistributifs au sein de chaque génération, entre ceux qui détiennent ces créances et ceux qui contribuent le plus, par l'impôt, au remboursement de la dette. Mais il s'agit là d'une problématique que W.S. Jevons n'évoque pas.

Le positionnement de W.S. Jevons à l'égard de la peur du déclin reste particulièrement pessimiste, dans la mesure où il ne prend pas la forme d'une solution radicale. Le principe de compensation intergénérationnelle, par le biais des infrastructures ou de la dette publique, ne constitue pas une solution à la problématique du déclin. Il ne s'agit que d'un contournement partiel du problème visant à assurer une situation de moindre mal aux générations futures, la question du déclin relatif par rapport à d'autres nations restant pleine et entière. 


\section{Régulations et confiance dans l'inventivité humaine}

Dans l'héritage de son père, H.S. Jevons reprend à son compte l'idée de la compensation intergénérationnelle par l'investissement en capital et dans l'éducation ([1915], p. 770). Une fois de plus, il ne s'arrête pourtant pas là, et décale légèrement la perspective de son aîné en donnant peu d'importance à la question de la dette publique. Cette orientation s'explique sans doute, là encore, par des évolutions historiques. Il apparaît que dès les années 1870 , à la suite de la politique fiscale menée par W.E. Gladstone, la dette publique en Grande-Bretagne a eu tendance à ralentir son rythme de croissance, voire à diminuer. La problématique de la double peine qui pouvait peser sur les générations à venir ne se pose donc plus selon les mêmes termes au moment où H.S. Jevons écrit. La proposition essentielle de ce dernier dans la gestion publique de l'industrie minière met en scène la question de la nationalisation de certaines ressources, ou de certains secteurs productifs. Pour H.S. Jevons, il existe des ressources naturelles qui apparaissent si rares que certains de leurs exploitants sont susceptibles de se retrouver en situation de monopole. C'est le cas, par exemple, de l'anthracite gallois ([1915], p. 325-326, 663). Pour éviter les dérives que pourrait provoquer une telle situation, c'est-à-dire pour éviter l'apparition de rentes de monopole qui provoqueraient un double renchérissement du minerai (épuisement + rente), H.S. Jevons propose une nationalisation de l'industrie minière galloise, ou du moins un contrôle strict par les autorités publiques de la gestion de l'anthracite, qui constitue un minerai stratégique dans le développement industriel britannique ([1915], p. 771).

Outre ces mesures de régulation assez radicales, H.S. Jevons développe des arguments substantiellement différents de ceux de son père sur la confiance qu'il est raisonnable de placer dans l'inventivité humaine pour relever les défis énergétiques à venir. Le recul historique dont il bénéficie n'est, à cet égard, pas anodin. Déjà avant lui, dès les années 1870, plusieurs observateurs refusaient de ne confier qu'au charbon le destin économique de la Grande-Bretagne. L'économiste John Marshall ([1878], p. 348) prenait l'exemple de la Hollande en soulignant que ses sous-dotations en ressources énergétiques n'étaient pas un obstacle à son développement économique. Le responsable politique et homme d'affaires Anthony J. Mundella
([1878], p. 89) fondait, quant à lui, la prospérité britannique sur deux piliers : un pilier énergétique certes, mais aussi un pilier humain, vantant la qualité de la main-d'œuvre et l'esprit d'entreprise des acteurs économiques de la Grande-Bretagne. Dans cette voie, H.S. Jevons nuance, lui aussi, le poids du charbon dans les potentiels à venir du développement britannique. Certes, l'énergie est une composante importante du prix des produits manufacturés, mais ces produits ne sont pas échangés et consommés uniquement sur un critère de prix. La sophistication du produit, sa capacité à répondre à de nouveaux besoins, sont des critères tout autant importants (H.S. Jevons [1915], p. 767768), surtout dans un contexte de concurrence entre nations où les consommateurs ont un pouvoir d'achat en hausse ${ }^{12}$.

L'accent mis ici par H.S. Jevons sur ce que nous pourrions nommer rétrospectivement des innovations de produit en appelle à la compétitivité hors-prix de l'industrie britannique, une compétitivité sur laquelle la Grande-Bretagne pourrait fonder son développement futur. Aux innovations de produit s'ajoutent des innovations organisationnelles : méthodes de travail, infrastructures logistiques, rapports entre unités de production et sous-traitants. Autant de domaines sur lesquels il serait possible d'agir pour assurer une perpétuation du développement. L'épuisement des ressources se retrouve relégué au second plan, comme H.S. Jevons l'admet lui-même :

I am, therefore, of opinion that under modern conditions of industry the question of whether or not we achieve the economies possible by improved organisation and by new inventions, due to the progress of science, is of far greater importance than the question of national disadvantage due to the exhaustion of our more cheaply workable coal supplies. ([1915], p. 767)

Remarquons que H.S. Jevons en appelle ainsi à un retour de la technique dans les solutions à apporter aux risques de déclin compétitif, mais par un biais inédit. Les nouveaux procédés ne sont pas à construire pour l'exploitation des minerais l'effet-rebond continuant à cet égard de jouer de

\footnotetext{
${ }^{12} \mathrm{Au}$ Royaume-Uni, les salaires réels ont augmenté de $45 \%$ entre 1880 et 1914 (Mitchell [1988], p. 150-151) (ratio calculé à partir des données de Mitchell). C'est l'aspect positif de l'augmentation des salaires, après son aspect plus négatif souligné par H.S. Jevons sur la question des coûts de la main-d'œuvre minière.
} 
mauvais tours ([1915], p. 722) - mais à d'autres fins, celles de mettre sur pied des produits manufacturés complexes qui, certes, seront plus onéreux, mais que les consommateurs seront prêts à payer pour leurs qualités propres. Dans l'appréhension de l'avenir économique britannique, l'optimisme prend alors le dessus sur le pessimisme, dans un contexte historique où le prix n'est plus la seule variable déterminante des échanges internationaux.

Ce relent d'optimisme chez H.S. Jevons s'explique également par les contraintes minières $\mathrm{du}$ début $\mathrm{du} \mathrm{XX}^{\mathrm{e}}$ siècle qui apparaissent plus souples que celles du milieu du XIX ${ }^{\mathrm{e}}$ siècle. Deux grandeurs l'illustrent, rapportées par H.S. Jevons lui-même ([1915], p. 787-788) : au milieu des années 1910, les réserves connues de charbon atteignent des niveaux sans comparaison avec les besoins des économies, 7397553 millions de tonnes à l'échelle de la planète, pour une consommation annuelle estimée à 1300 millions de tonnes. Même en supposant que les besoins soient amenés à croître, la marge de manœuvre semble bien grande, et le spectre de l'épuisement des ressources si lointain qu'il en deviendrait invisible.

\section{Conclusion}

Lorsqu'il entreprend en 1864-1865 son projet sur la question charbonnière, William Stanley Jevons le fait, selon ses propres mots ([1864], p. 52), avec un certain opportunisme. Dans une Grande-Bretagne victorienne prospère, où la Révolution industrielle a produit des effets économiques et démographiques sans précédent, les milieux intellectuels doutent de la pérennité d'un modèle à croissance exponentielle. L'analyse de W.S. Jevons fournit alors des arguments à celles et ceux qui craignent l'avènement d'un déclin économique, pour une nation qui est alors au sommet de la hiérarchie mondiale. Cinquante ans plus tard, en 1915, H.S. Jevons se fait l'écho des travaux de son père, cette fois-ci sans opportunisme. Professeur d'économie politique pendant plusieurs années à Cardiff, premier port mondial pour le commerce du charbon au tour- nant $\mathrm{du} \mathrm{XX}^{\mathrm{e}}$ siècle, il fait preuve d'une vraie érudition sur le sujet, en proposant une vaste monographie de la question charbonnière, non seulement sur le terrain des pratiques minières, mais aussi dans le domaine institutionnel et commercial.
Le diagnostic de ces deux auteurs sur la dépendance énergétique de l'appareil industriel britannique envers le charbon est sensiblement le même. Face à l'épuisement des ressources minières, le modèle de développement de la Grande-Bretagne ne peut perdurer, et il est à craindre que d'autres nations, en premier lieu les États-Unis, prendront le pas sur le Royaume-Uni à la tête de l'économie mondiale. C'est bien là un problème de déclin relatif qui est mis en évidence, selon des modalités diverses, directes et indirectes (perte de compétitivité, baisse des moyens financiers pour l'importation de denrées alimentaires), où l'épuisement des ressources joue un rôle crucial, même si H.S. Jevons met également en évidence d'autres facteurs déterminants. Éviter le déclin, ou du moins le rendre supportable, est une exigence qui répond à un souhait commun du père et du fils de donner une importance à la justice intergénérationnelle. En compensant les pertes futures par des investissements dans le présent, W.S. Jevons et H.S. Jevons proposent un principe fort qui n'est pas sans rappeler des débats plus récents sur la soutenabilité des appareils productifs. Parce que le contexte économique et énergétique dans lequel il écrit est différent de celui des années 1860 , H.S. Jevons élabore une analyse dont la modernité est saisissante. En insistant sur les dimensions organisationnelles et institutionnelles du développement industriel, il tend à indiquer que la réflexion en matière de ressources naturelles doit être une réflexion globale, où les procédés techniques doivent être associés à des choix politiques, le tout avec une prise en compte des réalités du commerce international. Autant de propositions qui reçoivent un important écho au $\mathrm{XXI}^{\mathrm{e}}$ siècle au sujet des régulations environnementales.

De la même façon, le pessimisme de W.S. Jevons n'est pas sans rappeler les craintes des années 1970 sur les limites de la croissance (voir, en particulier, Meadows et al. [1972]) : même avertissement au sujet de la finitude du monde, même appel à la solidarité intergénérationnelle, même scepticisme technique. Doit-on pour autant balayer d'un revers de la main les controverses du tournant $\mathrm{du}$ XXIe siècle au nom d'une simple résurgence historique qui en soulignerait la vacuité ? Probablement pas, car si les leçons de l'histoire sont utiles pour comprendre que le progrès technique est souvent sous-estimé, si elles permettent de mesurer la pertinence de certains 
discours contemporains mal à propos (à ce sujet, voir Missemer [2013]), elles restent parcellaires sur des sujets plus inédits comme celui qui est au cœur des enjeux contemporains, à savoir la question des changements climatiques. Recul historique et créativité sont donc appelés à aller de pair pour relever les défis d'aujourd'hui.

\section{Références bibliographiques}

BURAT A. [1869], Les Houillères en 1868, Paris, Hennoyer et fils.

CHURCH R. [1986], The History of the British Coal Industry, vol. 3, 1830-1913: The Victorian Pre-eminence, Oxford, Clarendon Press.

COPINSCHI P. [2012], Le Pétrole. Une ressource stratégique, Paris, La Documentation française.

COURNOT A.A. [1863], Principes de la théorie des richesses, dans Euvres complètes, vol. IX, édité par G. Jorland, 1981, Paris, Vrin et CNRS.

COURTNEY L.H. [1897], « Jevons's Coal Question: Thirty Years After, Royal Statistical Society », dans INOUE T. et MOSSELMANS B. (dir.), W. Stanley Jevons: Collected Reviews and Obituaries, vol. 1, Bristol, Thoemmes Press, 2002, p. 109-134.

FERNOW B.E. [1902], Economics of Forestry, New York, Crowell \& Co.

FLINN M.W. [1984], The History of the British Coal Industry, vol. 2, 1700-1830: The Industrial Revolution, Oxford, Clarendon Press.

FOUQUET R. [2009], «A Brief History of Energy », dans EVANS J. et HUNT L. C. (dir.), International Handbook on the Economics of Energy, Cheltenham \& Northampton, Edward Elgar Publishing, p. 1-19.

GALLOWAY R.L. [1882], A History of Coal Mining in Great Britain, Londres, MacMillan.

GOWDY J.M. et O’HARA S. [1997], «Weak Sustainability and Viable Technologies ", Ecological Economics, 22 (3), p. 239-247.
GREENING L.A., GREENE D.L. et DIFIGLIO C. [2000], « Energy Efficiency and Consumption The Rebound Effect - A Survey », Energy Policy, 28, p. 389-401.

HARTWICK J.M. [1977], « Intergenerational Equity and the Investing of Rents from Exhaustible Resources », American Economic Review, 67 (5), p. $972-974$.

HODGES I. [1910], « Increase of Working-Costs in Coal-Mines during the Past Half-Century, the Rate of Increase, and the Causes Thereof ", dans BENSON J. et OUTRAM Q. (dir.), Coal in Victorian Britain, part. 1, vol. 3, Londres, Pickering \& Chatto, 2012, p.141-148.

JEVONS H.S. [1905], Essays on Economics, Londres, MacMillan.

JEVONS H.S. [1915], The British Coal Trade, Londres, Norwich Press.

JEVONS W.S. [1864], « Letter to Herbert Jevons (18th February 1864) », dans BLACK R.D.C. (dir.), Papers and Correspondence of William Stanley Jevons, vol. 3, Londres, MacMillan, 1977, p. 52.

JEVONS W.S. [1866a], The Coal Question. An Inquiry Concerning the Progress of the Nation, and the Probable Exhaustion of our Coal Mines, Londres, MacMillan, $2^{\mathrm{e}}$ éd.

JEVONS W.S. [1866b], « Letter to Sir John F. W. Herschel (23 ${ }^{\text {rd }}$ June 1866) », dans BLACK R.D.C. (dir.), Papers and Correspondence of William Stanley Jevons, vol. 3, Londres, MacMillan, 1977, p. 122-123.

JEVONS W.S. [1867], « On Coal », dans BLACK R.D.C. (dir.), Papers and Correspondence of William Stanley Jevons, vol. 7, Londres, MacMillan, 1981, p. 18-28. Discours au Carpenters' Hall de Manchester, 16 janvier 1867.

JEVONS W.S. [1868], «On the Probable Exhaustion of Our Coal Mines ", dans BLACK R.D.C. (dir.), Papers and Correspondence of William Stanley Jevons, vol. 7, Londres, MacMillan, 1981, p. 28-35. Discours devant la Royal Institution, 13 mars 1868. 
JEVONS W.S. [1871], Theory of Political Economy, Londres, MacMillan. JEVONS W.S. [1875], " On the Progress of the Coal Question », dans BLACK R.D.C. (dir.), Papers and Correspondence of William Stanley Jevons, vol. 7, Londres, MacMillan, 1981, p. 36-37. Communication donnée à la British Association for the Advancement of Science.

MADDISON A. [2006], The World Economy, Paris, Presse de l'OCDE.

MARSHALL J. [1878], " The Coal Question. Continued », dans THORPE T.E. (dir.), Coal: Its History and Uses, Londres, MacMillan, p. 320-350.

MCCULLOCH J.R. [1830], Observations on the Duty on Sea-Borne Coal; and on the Peculiar Duties and Charges on Coal, Londres, Manning and Co.

MCCULLOCH J.R. [1835], " Philosophy of Manufactures », Edinburgh Review, 61, p. 453-472.

MEADOWS D.H., MEADOWS D.L., RANDERS J. et BEHRENS III W.W. [1972], The Limits to Growth, A Report for the Club of Rome's Project on the Predicament of Mankind, Londres, Potomac Associates.

MILL J.S. [1848], Principles of Political Economy, Boston (Mass.), Little \& Brown.

MISSEMER A. [2012], «William Stanley Jevons' The Coal Question (1865), beyond the Rebound Effect », Ecological Economics, 82, p. 97-103.

MISSEMER A. [2013], «William Stanley Jevons, un pionnier des réflexions sur la fiscalité écologique », L'Économie politique, 60, p. 78-90.

MISSEMER A. [2014], " L'analyse économique face à l'épuisement des ressources naturelles, de William Stanley Jevons à Harold Hotelling (18651931) - le cas des énergies fossiles ", Thèse de doctorat, Université de Lausanne et Université Lumière Lyon 2 (sous la direction de $\mathrm{P}$. Bridel et de N. Chaigneau).

MITCHELL B.R. [1988], British Historical Statistics, Cambridge (UK), Cambridge University
Press, Paperback Edition, 2011.

MUNDELLA A.J. [1878], «What are the Conditions on which the Commercial and Manufacturing Supremacy of Great Britain Depend, and is there any Reason to think they have been, or may be, Endangered? », Journal of the Statistical Society of London, 41(1), p.87-134.

NEUMAYER E. [1999], Weak versus Strong Sustainability. Exploring the Limits of Two Opposing Paradigms, Cheltenham and Northampton, Edward Elgar Publishing.

OUTRAM Q. [2012a], "Costs and Efficiency », dans BENSON J. et OUTRAM Q. (dir.), Coal in Victorian Britain, part. 1, vol. 3, Londres, Pickering \& Chatto, p. 107-115.

OUTRAM Q. [2012b], "Introduction », dans BENSON J. et OUTRAM Q. (dir.), Coal in Victorian Britain, part. 1, vol. 3, Londres, Pickering \& Chatto, p. xi-xix.

OUTRAM Q. [2012c], « Organization », dans BENSON J. et OUTRAM Q. (dir.), Coal in Victorian Britain, part. 1, vol. 2, Londres, Pickering \& Chatto, p. 375-378.

OUTRAM Q. et FISHMAN N. [2006], " Le roi Charbon et ses sujets turbulents : les industries et sociétés charbonnières en Grande-Bretagne aux $\mathrm{XVIII}^{\mathrm{e}}$ et $\mathrm{XIX}^{\mathrm{e}}$ siècles ", dans DAUMALIN X., DAVIET S. et MIOCHE P. (dir.), Territoires européens $d u$ charbon des origines aux reconversions, Aix-en-Provence, Publications de l'Université de Provence, p. 15-35.

PASSET R. [2010], Les Grandes Représentations du monde et de l'économie à travers l'histoire, Paris, Les Liens qui libèrent.

PEARCE D.W. et ATKINSON G.D. [1993], « Capital Theory and the Measurement of Sustainably Development: an Indicator of "Weak" Sustainability », Ecological Economics, 8 (2), p. 103-108.

PERCY C.M. [1888], Mine Rents and Mineral Royalties, Liverpool, Financial Reform Association. 
RICARDO D. [1821], The Principles of Political Economy and Taxation, Londres et New York, Dent \& Dutton [éd. 1911].

ROBINE M. [1990], « La question charbonnière de William Stanley Jevons ", Revue économique, 41 (2), p. 369-394.

SAY J.-B. [1828], Cours complet d'économie politique pratique, t. 2, Paris, Rapilly.

SENIOR N.W. [1836], An Outline of the Science of Political Economy, New York,
Augustus M. Kelley [éd. 1965].

SIMONIN L. [1867], La Vie souterraine ou les mines et les mineurs, Paris, Hachette. WHITE M.V. [1991], «Where Did Jevons' Energy Come From? », History of Economics Review, 15, p. 6072.

WHITE M.V. [2004], «In the Lobby of the Energy Hotel: Jevons's Formulation of the Postclassical "Economic Problem" », History of Political Economy, 36 (2), p. 227-271. 